\title{
The additional value of an oblique image plane for MRI of the anterior and posterior distal tibiofibular syndesmosis
}

\author{
John J. Hermans • Abida Z. Ginai • Noortje Wentink • \\ Wim C. J. Hop • Annechien Beumer
}

Received: 13 July 2009 /Revised: 12 March 2010 /Accepted: 12 April 2010 / Published online: 13 June 2010

(C) The Author(s) 2010. This article is published with open access at Springerlink.com

\begin{abstract}
Objective The optimal MRI scan planes of collateral ligaments of the ankle have been described extensively, with the exception of the syndesmotic ligaments. We assessed the optimal scan plane for depicting the distal tibiofibular syndesmosis.

Materials and Methods In order to determine the optimal oblique caudal-cranial and lateral-medial MRI scan plane, two fresh frozen cadaveric ankles were used. The angle of the scan plane that demonstrated the anterior and posterior distal tibiofibular ligament uninterrupted in their full length was determined. In a prospective study this oblique scan plane was then used in addition to the axial and coronal planes, for MRI scans of both ankles in 21 healthy volunteers. Two observers independently evaluated the anterior tibiofibular ligament (ATIFL) and posterior tibiofibular ligament (PTIFL) regarding the continuity of the individual fascicles, thickness and wavy contour of the
\end{abstract}

Electronic supplementary material The online version of this article (doi:10.1007/s00256-010-0938-9) contains supplementary material, which is available to authorized users

J. J. Hermans $(\varangle) \cdot$ A. Z. Ginai $\cdot$ N. Wentink

Department of Radiology, Erasmus University Medical Center,

PO Box 2040, 3000 CA Rotterdam, The Netherlands

e-mail: jjhermans@gmail.com

W. C. J. Hop

Department of Epidemiology and Biostatistics,

Erasmus University Medical Center,

PO Box 2040, 3000 CA Rotterdam, The Netherlands

A. Beumer

Department of Orthopaedics, Upper Limb Unit,

Amphia Ziekenhuis,

PO Box 90158, 4800 RK Breda, The Netherlands ligaments in both the axial and the oblique plane. Kappa was calculated to determine the interobserver agreement. McNemar's test was used to statistically quantify the significance of the two scan planes.

Results In the axial plane the ATIFL was in 31\% (13/42) partly and in 69\% (29/42) completely discontinuous; in the oblique plane the ATIFL was continuous in $88 \%(37 / 42)$ and partly discontinuous in $12 \%(5 / 42)$. Compared with the axial plane, the oblique plane demonstrated significantly less discontinuity $(p<0.001)$, but not significantly less thickening ( $p=1.00)$ or less wavy contour $(p=0.06)$ of the ATIFL. In the axial scan plane the PTIFL was continuous in $76 \%(32 / 42)$, partially discontinuous in $19 \%(8 / 42)$ and completely discontinuous in $5 \%(2 / 42)$; in the oblique plane the PTIFL was continuous in $100 \%(42 / 42)$. Compared with the axial plane, the oblique plane demonstrated significantly less discontinuity $(p=0.002)$, but not significantly less thickening $(p=1.00)$ or less wavy contour $(p=0.50)$ of the PTIFL. The interobserver agreement score and kappa ( $\mathrm{K}$ ) regarding the continuity for the ATIFL in the axial and oblique planes was $91 \%(\kappa=0.79)$ and $91 \%$ $(\kappa=0.55)$ respectively; for the PTIFL it was $86 \%(\kappa=0.65)$ and $100 \%$ ( $K=$ not defined).

Conclusion The ATIFL and PTIFL are routinuely scanned in the orthogonal planes. The advantage of MRI scanning in an oblique image plane of about 45 degrees permits a better evaluation of the ligaments compared with the axial plane, particularly a better interpretation of ligament continuity, thickening and wavy contour. This may lead to a reduction in false-positive results, especially regarding partial or complete ligament ruptures. This can be of considerable aid in therapeutic management.

Keywords Ankle · MRI · Oblique scan · Tibiofibular syndesmosis 


\section{Abbreviations}

ATIFL Anterior tibiofibular ligament

PTIFL Posterior tibiofibular ligament

\section{Introduction}

Injury of the distal tibiofibular syndesmosis occurs in approximately $1-11 \%$ of all ankle sprains $[1-3]$. This number may increase to more than $40 \%$ in those actively involved in high contact or collision sporting activities [4].

Syndesmotic instability in adults can be due to widening of the ankle mortise (complete tibiofibular diastasis) or to posterior translation and external rotation of the fibula (anterior tibiofibular diastasis). The latter is more common and more difficult to recognize. The chronic instability results from increased length of the syndesmotic ligaments that have healed and elongated after an acute rupture that was not adequately treated [5-7]. Lateral shift of the talus more than $2 \mathrm{~mm}$ and external rotation of the talus more than $5^{\circ}$ reduce the mean joint contact area and increase the contact pressure in the ankle $[8,9]$. Since widening of the ankle mortise by $1 \mathrm{~mm}$ decreases the contact area of the tibiotalar joint by $42 \%$ [10] this could lead to early osteoarthritis of the tibiotalar joint.

Radiography, arthrography, ultrasound, and CT have been used to assess the integrity of the syndesmosis. Several authors have described that radiography has limited use in the assessment of syndesmotic integrity [11-13]. Conventional $\mathrm{X}$-ray arthrography is not routinely used anymore as it is an invasive examination, although it clearly displays syndesmotic and other ligamentous injuries [14-16]. Ultrasound can clearly depict a torn tibiofibular ligament in the acute stage but is less accurate over time and the results are very dependent on the skills of the radiologist [17]. With the use of CT, widening of the mortise exceeding $3 \mathrm{~mm}$ can be reliably assessed, as well as gross deformity [18]. However, external rotation of the distal fibula, such as that seen in anterior tibiofibular diastasis, is not easily recognized, because of the "round" shape of the fibula at that level.

The value of MRI in acute and chronic syndesmotic injuries has been described in several papers [19-23]. In these papers the usual three orthogonal scan planes (axial, coronal, and sagittal) and the position of the foot during imaging (neutral, $10-20^{\circ}$ dorsiflexion or $40-50^{\circ}$ plantar flexion) have been used to evaluate the optimal scan plane of both the collateral and the syndesmotic ankle ligaments $[21,23]$. However, since the anterior and posterior distal tibiofibular ligaments run obliquely to the orthogonal planes [24], the axial scan plane may lead to a false-positive interpretation regarding the presence of a syndesmotic injury when MRI results are compared with operative findings $[20,25]$.
In the present study we assessed the additional value of an oblique scan plane for depicting the anterior and posterior distal tibiofibular syndesmotic ligaments in cadaveric specimens and validated this in healthy volunteers. The clinical relevance is illustrated with three clinical cases.

\section{Materials and methods}

In two fresh frozen cadaveric lower legs, amputated below the knee, we obtained images of the ankle in a $1.5 \mathrm{~T}$ MR (Philips Gyroscan, Best, The Netherlands). The legs were thawed for $24 \mathrm{~h}$ before scanning. We used a wrap-around surface coil (E1 coil). A T1-weighted turbo spin echo (TSE) sequence was acquired (TR $500 \mathrm{~ms}$; TE $15 \mathrm{~ms}$; TF 5) with a FOV of $12 \times 12 \mathrm{~cm}$, a matrix of $512 \times 512$ and slice thickness of $2.5 \mathrm{~mm}$ with a gap of $0.2 \mathrm{~mm}$ and NSA of 2 .

The images were acquired in the axial, coronal, sagittal, and several oblique planes, with the foot in the neutral position. The oblique image plane was defined in the coronal and sagittal plan scans. In the coronal view the acute angle of the oblique plane was related to the tibial plafond and ran caudal-cranially and lateralmedially through the distal fibula. The angle of the oblique plane varied from $30-60^{\circ}$ with steps of $5^{\circ}$ (Fig. 1a). In the sagittal view the direction of the oblique plane ran parallel to a line along the inferior border of the anterior and posterior tibia (Fig. 1b). The angle of the scan plane that demonstrated the multiple fascicles of the anterior (ATIFL) and posterior distal tibiofibular ligaments (PTIFL) uninterrupted in their full length was defined as the optimal angle. The optimal angle was defined by the consensus of two observers ( $\mathrm{JH}, \mathrm{NW}$ ).

After determination of the optimal angle of the oblique MRI scan plane, this plane was used in addition to the axial and coronal views to scan the syndesmosis of both ankles in 21 healthy volunteers (9 men, range 21-30 years, and 12 women, range 21-30 years). Inclusion criteria for the volunteers were: age over 18 years, no history of trauma in the past year, no previous surgery and no inflammatory disease of the ankle or foot. The same MR scanner and T1weighted TSE sequences as in the cadaveric legs were applied. The anterior and posterior distal tibiofibular ligaments were evaluated with respect to continuity of the separate fascicles, thickening of the fascicles, and a wavy contour of the ligament [26]. Regarding the continuity of the fascicles the ATIFL and PTIFL were assigned a score in both the axial and the oblique image planes: "zero" when none of the fascicles of the ligament between the tibia and fibula was interrupted (i.e., continuous), "one" when one or more, but not all of the fascicles, were interrupted (i.e., partly interrupted), and "two" when all fascicles were interrupted (i.e., completely interrupted). Thickening of 
Fig. 1 a Coronal and b sagittal MR images, which indicate the angle of the oblique image plane for the anterior and posterior distal tibiofibular ligaments. In the coronal plane the angle varied from 35 to $60^{\circ}$, with steps of $5^{\circ}$. In the sagittal view the direction of the oblique plane runs parallel to a line along the inferior border of the anterior and posterior tibia
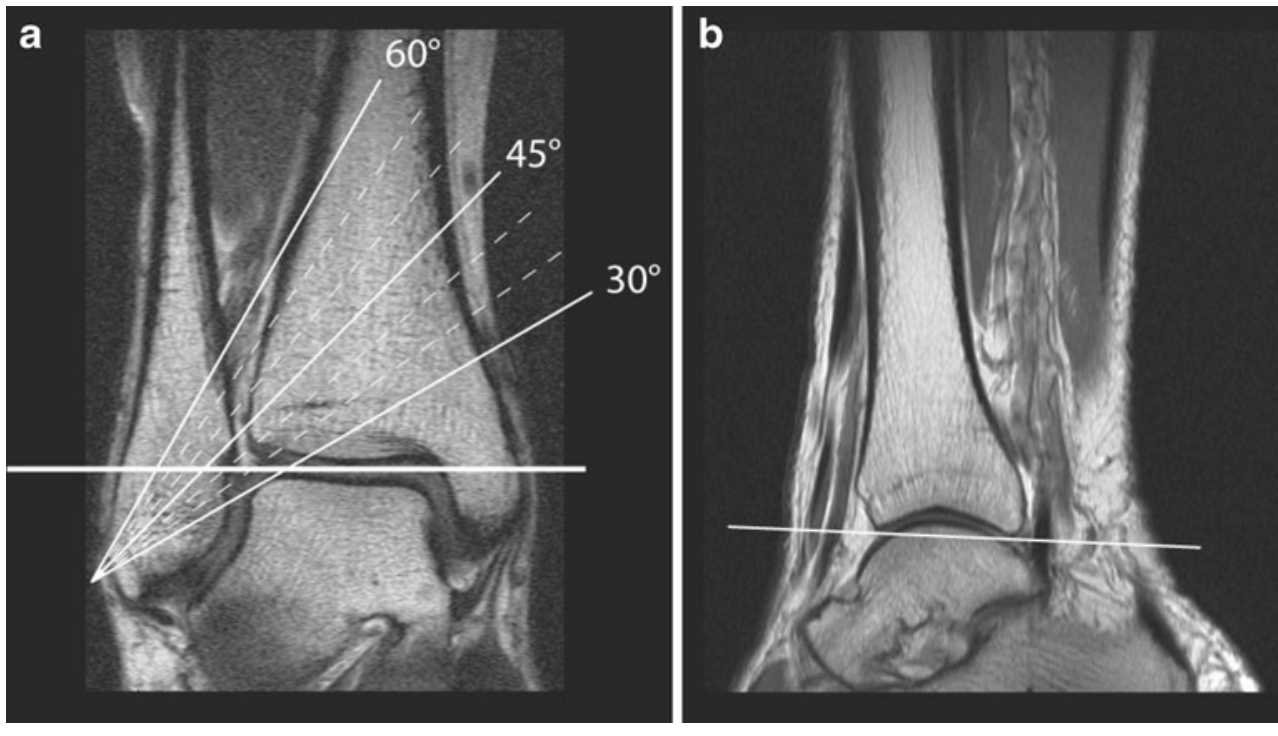

the fascicles and a wavy contour of the ligament were scored as either present or absent.

The images were independently analyzed by two musculoskeletal radiologists with $10(\mathrm{JH})$ and 30 (AG) years' experience. The interobserver agreement was determined by the kappa agreement score $(0.00-0.20$ : poor; 0.21-0.40: fair; $0.41-0.60$ moderate; $0.61-0.80$ good; 0.81-0.90: very good; 0.91-1.00: extra good). McNemar's test was used to statistically quantify the significance of the axial and oblique scan planes regarding the continuity, thickening and wavy contour of the anterior and posterior distal tibiofibular ligaments, with a $p$ value less than 0.05 considered significant. Statistical analysis was performed with Statistical Package for Social Sciences (SPSS) 15.0.

In three clinical cases the additional value of the oblique scan plane was demonstrated with respect to diagnosis and treatment.

\section{Results}

Cadaveric ankles

When the ankle joint was imaged in the axial plane the fascicles of the anterior distal tibiofibular ligament appeared to be discontinuous between the anterior tubercle of the tibia and the anterior tubercle of the fibula. This apparent discontinuity slowly decreased when the $30^{\circ}$ angle of the scan plane was increased by steps of $5^{\circ}$. At about $35^{\circ}$ the uninterrupted ATIFL became visible and showed the multifascicular aspect of the ligament to good advantage. With a scan angle greater than $55^{\circ}$ the fascicles appeared to be discontinuous again. The plane of $45^{\circ}$ also demonstrated the posterior distal tibiofibular ligament in its full length. In both the coronal and sagittal planes the ATIFL and PTIFL were not depicted with as much detail as in the oblique plane.

\section{Volunteers}

The interobserver agreement was calculated with kappa and the agreement score (Table 1).

The axial scan plane demonstrated partial (Fig. 2) or complete discontinuity (Fig. 3) of the ATIFL in 31\% (13 out of 42 ) and $69 \%$ (29 out of 42 ) of the 21 volunteers respectively. In the $45^{\circ}$ oblique scan plane the ATIFL was continuous in $88 \%$ (37 out of 42 ) and partially discontinuous in $12 \%$ ( 5 out of 42 ). In the oblique plane the fascicles were more often continuous than in the axial plane $(p<$ 0.001). When compared with the axial plane, the oblique plane demonstrated less thickening $(5 \%$ and $2 \%$ respec-
Table 1 Agreement score $(A S$ [\%]) and kappa (K), regarding continuity, thickening, and wavy contour of the anterior tibiofibular ligament (ATIFL) and posterior tibiofibular ligament $(P T I F L)$ in the axial and oblique planes of both ankles in 21 volunteers

\begin{tabular}{|c|c|c|c|c|c|c|c|c|}
\hline & \multicolumn{4}{|l|}{ ATIFL } & \multicolumn{4}{|l|}{ PTIFL } \\
\hline & \multicolumn{2}{|l|}{ Axial } & \multicolumn{2}{|l|}{ Oblique } & \multicolumn{2}{|l|}{ Axial } & \multicolumn{2}{|l|}{ Oblique } \\
\hline & AS $(\%)$ & K & AS (\%) & K & AS (\%) & K & AS (\%) & K \\
\hline Continuity & 91 & 0.79 & 91 & 0.55 & 86 & 0.65 & 100 & Not determined \\
\hline Thickening & 91 & 0.46 & 95 & 0.48 & 95 & 0.72 & 98 & 0.79 \\
\hline Wavy & 91 & 0.77 & 98 & 0.90 & 88 & 0.23 & 95 & Not determined \\
\hline
\end{tabular}


Fig. 2 a Axial and b oblique T1-weighted turbo spin echo (TSE) image of the anterior distal tibiofibular ligament in a healthy volunteer. In the axial image (a) two of the three visible fascicles of the anterior tibiofibular ligament (ATIFL) are partially interrupted (arrow). In the oblique image (b) all three fascicles are continuous and visible along their entire length. Therefore, partial discontinuity of the fascicles of the ATIFL cannot be used as a parameter for a partial rupture of the anterior syndesmosis, when scanned in an axial plane
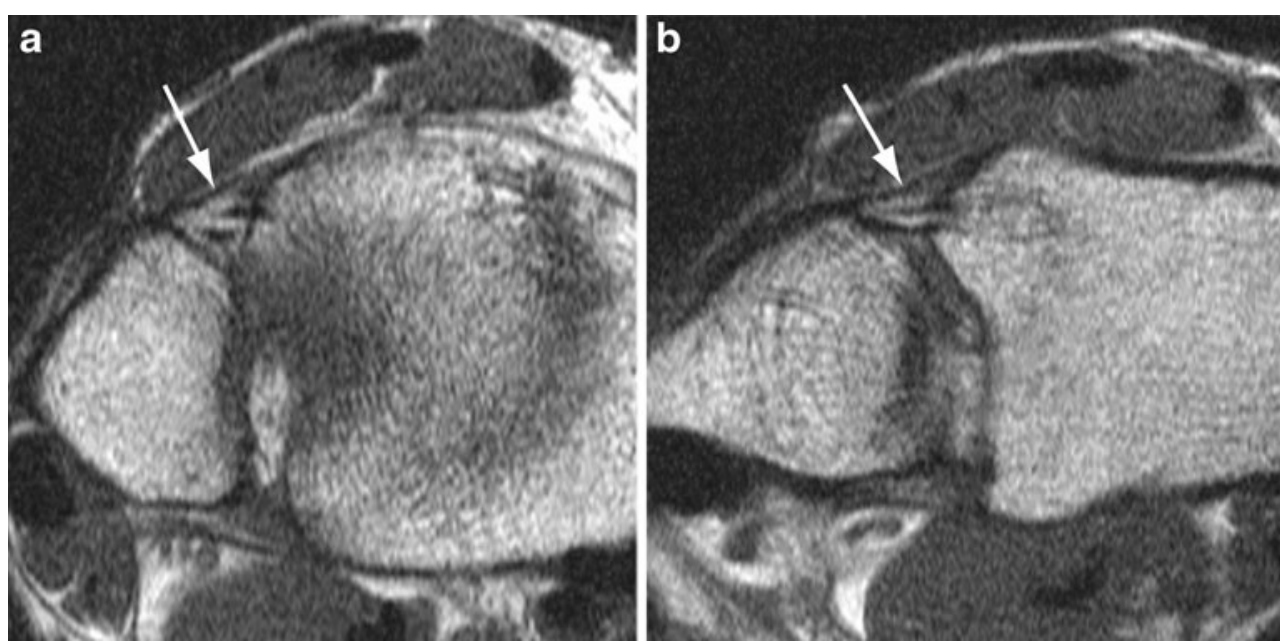

tively; $p=1.00)$ and less wavy contour (24\% and $12 \%$ respectively; $p=0.06$ ) of the ATIFL, but neither was significant (Table 2).

In the axial scan plane the PTIFL was continuous in $76 \%$ (32 out of 42 ), partially discontinuous in $19 \%$ (8 out of 42 ) and completely discontinuous in $5 \%$ (2 out of 42 ). In the oblique plane the PTIFL was continuous in $100 \%$ (42 out of 42; Fig. 4). In the oblique plane the fascicles were more often continuous than in the axial plane $(p=0.002)$. When compared with the axial plane, the oblique plane demonstrated less thickening (10\% and $7 \%$ respectively; $p=1.00)$ and less wavy contour $(5 \%$ and $0 \%$ respectively; $p=0.50$ ) of the PTIFL, but neither was significant (Table 2).

\section{Clinical cases}

The clinical relevance of the oblique scan plane for the syndesmosis is demonstrated with three cases. The first case was a 37-year-old patient with a sprained ankle (Fig. 5). On the T2-weighted turbo spin echo (TSE) images in the axial plane the ATIFL appeared discontinuous. In the oblique scan plane, however, the anterior syndesmosis was continuous. Therefore, the ATIFL was not completely ruptured, as suggested by the image in the axial plane. Although an isolated rupture of the ATIFL can be treated non-operatively, it should preferably be treated in plaster, whilst a sprain, i.e., a partial rupture of the anterior syndesmosis, can be treated functionally [27]. An additional finding of interest was a bone bruise of the distal tibia as indicated by the high signal intensity area on the coronal short tau inversion recovery (STIR) image (Fig. 5c).

The second case was a 44-year-old man who complained of chronic instability after he had sprained his ankle about a year ago (Fig. 6). The T1-weighted turbo spin echo (TSE) axial image showed a thickened and discontinuous aspect of the ATIFL, while on the oblique image the ATIFL was continuous. The anterior syndesmosis was thickened

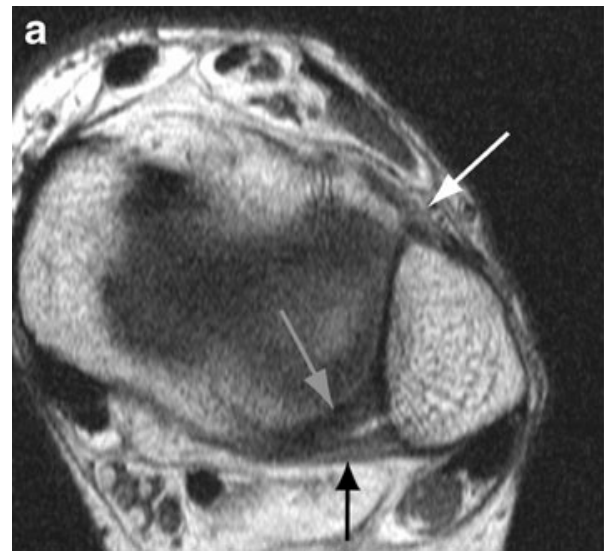

Fig. 3 a Axial and $\mathbf{b}$ oblique T1-weighted TSE image of the anterior distal tibiofibular ligament in a healthy volunteer. The axial image (a) shows a discontinuous aspect of the inferior fascicle of the ATIFL, also called Bassett's ligament (white arrow). In the oblique image (b)

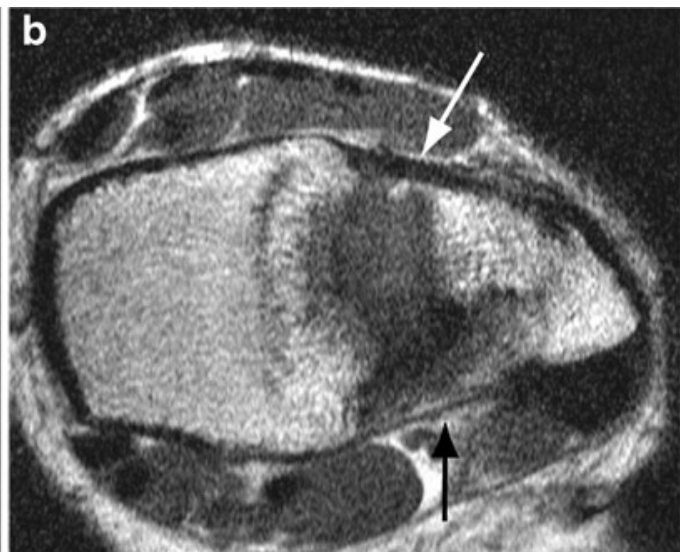

a thick continuous Bassett's ligament is visible. Posteriorly the transverse ligament (gray arrow) and the posterior tibiofibular ligament (PTIFL; black arrow) are depicted; in the oblique plane (b) only the lowermost fascicle of the PTIFL is depicted at this level 
Table 2 Frequencies of ligament characteristics of ATIFL and PTIFL in the axial and oblique planes of both ankles in 21 volunteers. The fascicles are continuous $(\mathrm{CO})$, or partially or completely interrupted $(P I$ or $C I)$

\begin{tabular}{|c|c|c|c|c|c|c|c|c|}
\hline \multirow[t]{2}{*}{ Axial \oblique } & \multicolumn{4}{|c|}{ ATIFL } & \multicolumn{4}{|c|}{ PTIFL } \\
\hline & $\mathrm{CO}$ & PI & $\mathrm{CI}$ & Total & $\mathrm{CO}$ & PI & CI & Tota \\
\hline $\mathrm{CO}$ & 0 & 0 & 0 & 0 & 32 & 0 & 0 & 32 \\
\hline PI & 12 & 0 & 1 & 13 & 8 & 0 & 0 & 8 \\
\hline \multirow[t]{2}{*}{ CI } & 25 & 0 & 4 & 29 & 2 & 0 & 0 & 2 \\
\hline & 37 & 0 & 5 & 42 & 42 & 0 & 0 & 42 \\
\hline
\end{tabular}

because of fibrosis, which makes the fascicles of the ligament no longer individually visible. In the chronic situation an anatomical reconstruction is the best treatment if the ATIFL is continuous. If not, salvage surgery such as tenodesis or arthrodesis may be performed. These surgeries relieve the instability, but alter the biomechanics [5].

The third case was a 12-year-old boy with an acute sprain of his left ankle (Fig. 7). He had sustained a sprain of the same ankle 8 months previously. Extensive imaging (X-ray, $\mathrm{CT}$, and MRI) finally demonstrated an old avulsion fracture of the anterior tubercle of the distal fibula (WagstaffeLefort). The oblique plane demonstrated an intact anterior distal tibiofibular ligament attached to an avulsion fracture of the anterior fibula, whereas axial images at adjacent levels of the distal tibiofibular joint showed the avulsion fracture of the anterior aspect of the fibula with a discontinuous aspect of the ATIFL. Based on this information the surgeon could fix the avulsed fibular fragment with a staple and stabilize the syndesmosis with a setscrew, after debridement of the medial gutter to allow the talus to resume its normal position. Without the information obtained from the oblique MRI plane, a more substantial reconstructive surgery would have been planned.

\section{Discussion}

Many studies have been carried out to determine the optimal plane for scanning the lateral collateral ankle ligaments with MRI. Only a few studies have been performed regarding the optimal scan plane for the syndesmotic ligaments $[21,23]$. According to the literature the axial and coronal scan planes are optimal for depicting the ATIFL, PTIFL, and transverse ligaments, and the axial plane for the interosseous ligament. The basic concept is that a ligament is depicted best when the scan plane runs parallel to the direction of that ligament. Since the ATIFL and PTIFL run in an oblique direction with respect to the tibial plafond, imaging of the ATIFL and PTIFL should be optimal in an oblique plane, instead of the commonly used axial and coronal planes. As one can expect, an obliquely running ligament will likely show a partial or complete discontinuity when imaged in an axial plane.

The ATIFL has anteroposteriorly a triangular shape, is about $1 \mathrm{~cm}$ in height and has a thickness of $5 \mathrm{~mm}$. It consists of three bundles, separated by 2-mm wide gaps that slightly converge in the laterodistal direction [24]. The superficial anterior fibers are $2-3 \mathrm{~cm}$ long; the deeper posterior fibers are somewhat shorter [3]. It extends in an oblique way between the anterior tubercle of the distal tibia and the anterior tubercle of the distal fibula and runs from medial-superior to lateral-inferior and crosses the anterolateral corner of the talus. The angle formed between a line along the tibial plafond in the coronal view and a line along the ATIFL varies from 30 to $50^{\circ}[24,28-30]$.

The PTIFL has a quadrilateral shape, is about $1 \mathrm{~cm}$ in height, $6 \mathrm{~mm}$ thick and $18 \mathrm{~mm}$ wide. Its fibers extend between the posterior tibial malleolus and the posterior tubercle of the fibula from medial-superior to lateralinferior. It runs slightly more horizontally than the ATIFL [24].
Fig. 4 a Axial and b oblique T1-weighted TSE image of the posterior distal tibiofibular ligament (arrow) in a healthy volunteer. In the axial image (a) the discontinuous fascicles of the PTIFL are visible, whilst in the oblique image (b) the fascicles are intact. Therefore, in the oblique image plane the posterior syndesmosis is demonstrated to its better advantage
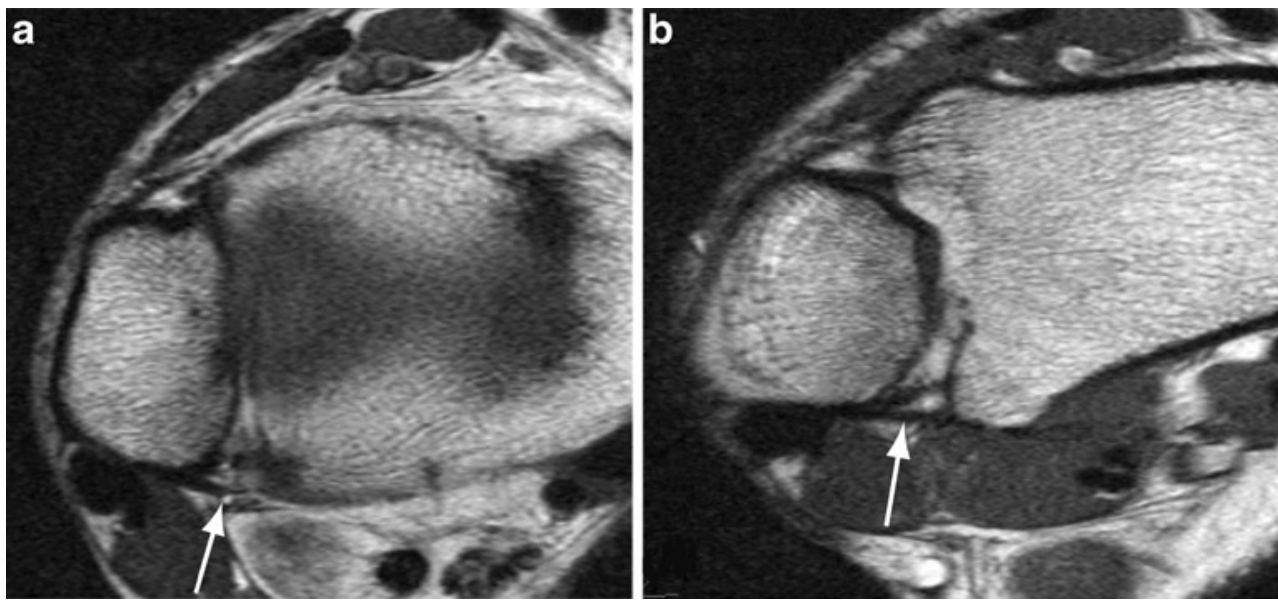

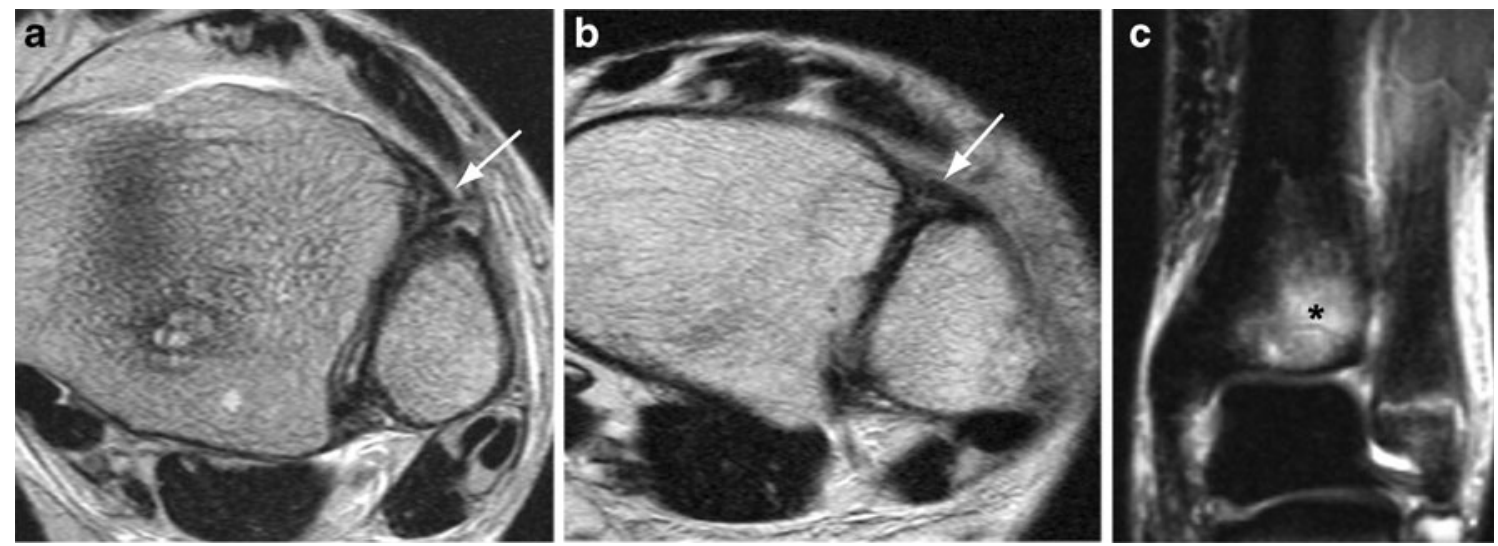

Fig. 5 a Axial and b oblique T2-weighted TSE image of an ankle sprain. The ATIFL (arrow) is discontinuous on the axial image (a) and, although a little thickened, the ligament is continuous on the oblique image (b). The anterior syndesmosis is therefore not ruptured

Based on these data it seemed a logical step to introduce an oblique image plane for depicting the ATIFL and PTIFL. To find the optimal angle of this oblique plane we first used two cadaveric lower legs. We increased the angle gradually from 30 to $60^{\circ}$ in steps of $5^{\circ}$. In the range of $35-55^{\circ}$, the individual fascicles of both the ATIFL and PTIFL were visible in their entire length. We finally chose an angle of $45^{\circ}$ (in consensus $\mathrm{JH}, \mathrm{NW}$ ) as the optimal angle for the oblique image plane, as this angle has an upper and lower range of about $10^{\circ}$ without the risk of not depicting the ligaments properly.

In our volunteers we analyzed the ATIFL and PTIFL with regard to continuity, thickening, and a wavy aspect of the ligament, since these are characteristics of ligamentous injury [26]. In the axial plane the ATIFL and PTIFL were continuous in $0 \%$ and $76 \%$ respectively, while they were continuous in $88 \%$ and $100 \%$ respectively in the oblique plane. In the axial plane the fascicles were depicted as partly or completely interrupted. This could easily give the impression of a rupture of the ligament [25]. An example of this problem is demonstrated in the paper by Oae et al. [25], as suggested by the axial image. The coronal short tau inversion recovery (STIR) image shows bone marrow edema in the distal tibia (asterisk, c)

where axial MRI images showed a rupture of the ATIFL in one patient and a rupture of the PTIFL in another, both of which proved to be incorrect at ankle arthroscopy. Since the range in the angle of the anterior distal tibiofibular ligament varies, not all of its fascicles will always lie in the oblique image plane of $45^{\circ}$, as occurred in $12 \%$ of the healthy volunteers.

Other characteristics of an injured ligament are thickening and a wavy aspect. In two volunteers, one or more of the fascicles of the ATIFL appeared to be thickened in the axial plane (in only one in the oblique plane). Bassett et al. described a thick inferior fascicle of the ATIFL as a possible cause of anterolateral tibiotalar impingement [31]. The ATIFL consisted of two or three rather thick fascicles in some, and multiple thin fascicles in other volunteers. Since none of the volunteers had a history of serious ankle trauma or current ankle complaints, it is reasonable to assume that a thickened aspect of one or more of the fascicles, especially when present in both ankles, could be a normal variant. Possibly, the ATIFL is composed of a variable number of fascicles, with variable thickness,
Fig. 6 a Axial and b oblique T1-weighted TSE image of a chronic injury of the ATIFL. The axial image shows a thickened and discontinuous aspect of the ATIFL (white arrow, a). However, in the oblique image the ligament is continuous, although quite thickened (white arrow, b). The two fascicles of the PTIFL are continuous, but slightly thickened in both the axial and oblique planes (black arrow)
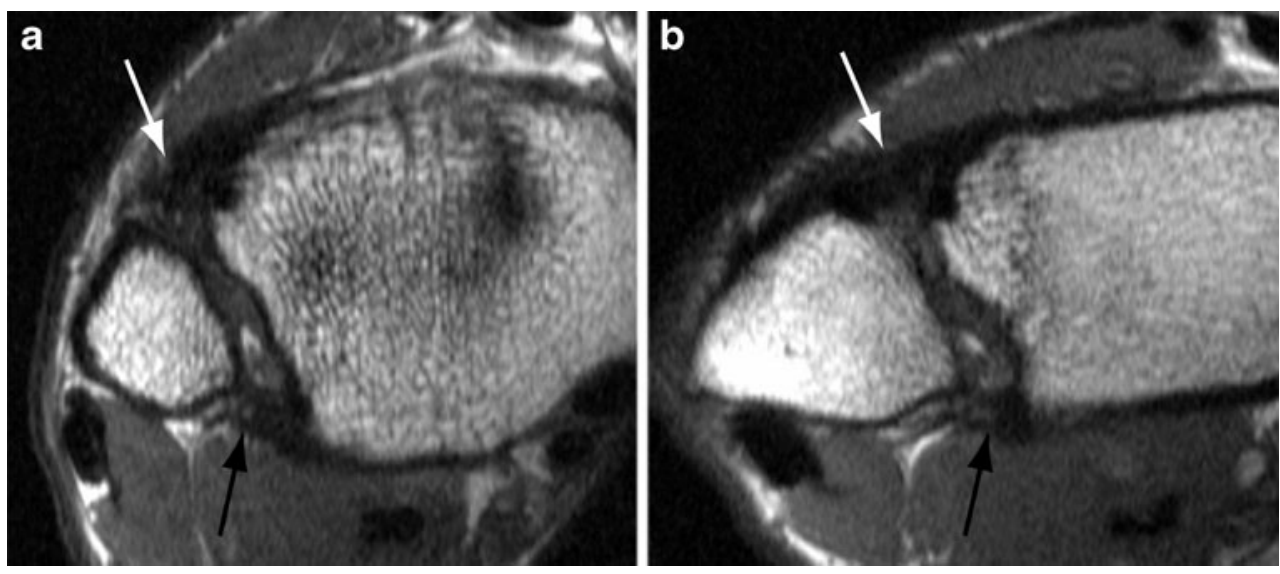
Fig. 7 a, b Axial and $\mathbf{d}$ oblique proton density and $\mathbf{c}$ axial

T2-weighted TSE images of a 12-year-old boy. The two axial proton density-weighted images, at two adjacent levels of the distal tibiofibular joint (a, b), demonstrate the avulsion fracture of the anterior aspect of the fibula (Wagstaffe-Lefort, asterisk), with a discontinuous aspect of the anterior distal tibiofibular ligament (arrow). The axial T2-weighted image (c) also shows the fibular avulsion fracture and interrupted anterior syndesmosis. However, on the oblique proton density image (d) the fibular avulsion fragment (asterisk) is attached to an intact anterior distal tibiofibular ligament (arrow)
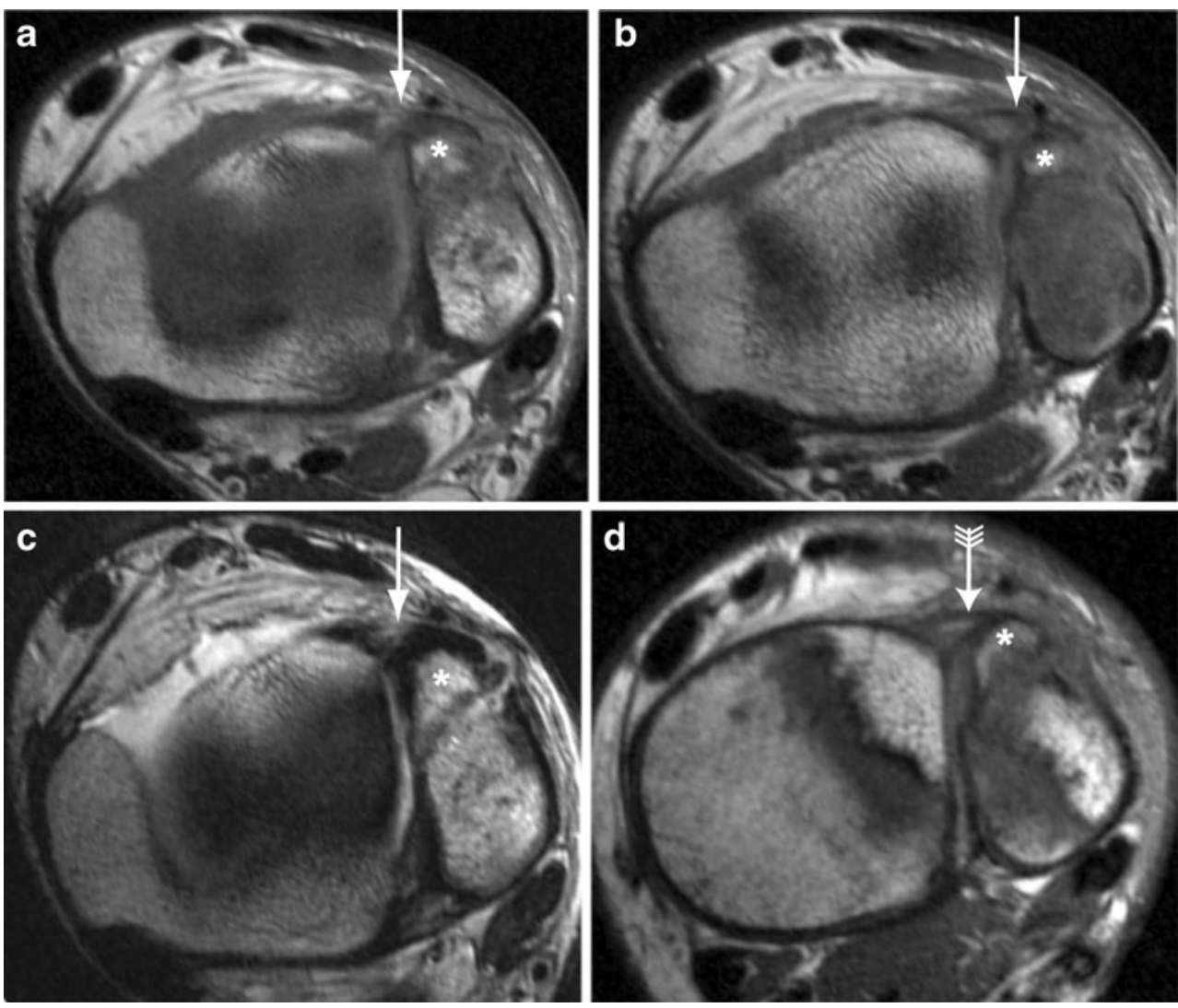

instead of the three mentioned by Bartonicek [24]. Subhas et al. also mention a thickened inferior ligament (i.e., Bassett's) in the presence of a normal ATIFL [32].

In a few cases the ATIFL or PTIFL demonstrated a wavy aspect in the axial plane, in 10 and 2 cases respectively, and remained wavy in the oblique plane, in 2 and 0 cases respectively (Fig. 8). It is not clear why the wavy aspect of these ligaments, as observed in the axial plane, disappeared in the oblique plane. Possibly, insertion of the ligament onto a slightly protruding tibial and fibular tubercle gives this impression. A wavy aspect of the syndesmotic ligaments has until now been interpreted as a sign of injury and could therefore also lead to false-positive findings.

Although the agreement score regarding the different characteristics of the ATIFL and PTIFL was generally good (86-100\%), the kappa value (K), however, varied from fair to good (0.23-0.90; Table 1). For example, the agreement score regarding continuity of the PTIFL in the oblique plane was $100 \%$, while kappa was only 0.65 . Discrepancies between the percentage agreement and kappa values in our case can be explained by the skewed distribution of categories. In such cases kappa is known to have poor
Fig. 8 a Axial and b oblique T1-weighted TSE image of the ATIFL (arrow) in a healthy volunteer. The ATIFL has a wavy aspect on the axial image (a) and a straight aspect in the oblique image (b). The continuity and multifascicular aspect of the ATIFL are better demonstrated on the oblique image. A wavy aspect of the ATIFL can thus not be used as a parameter for injury of the ATIFL, in an axial scan plane
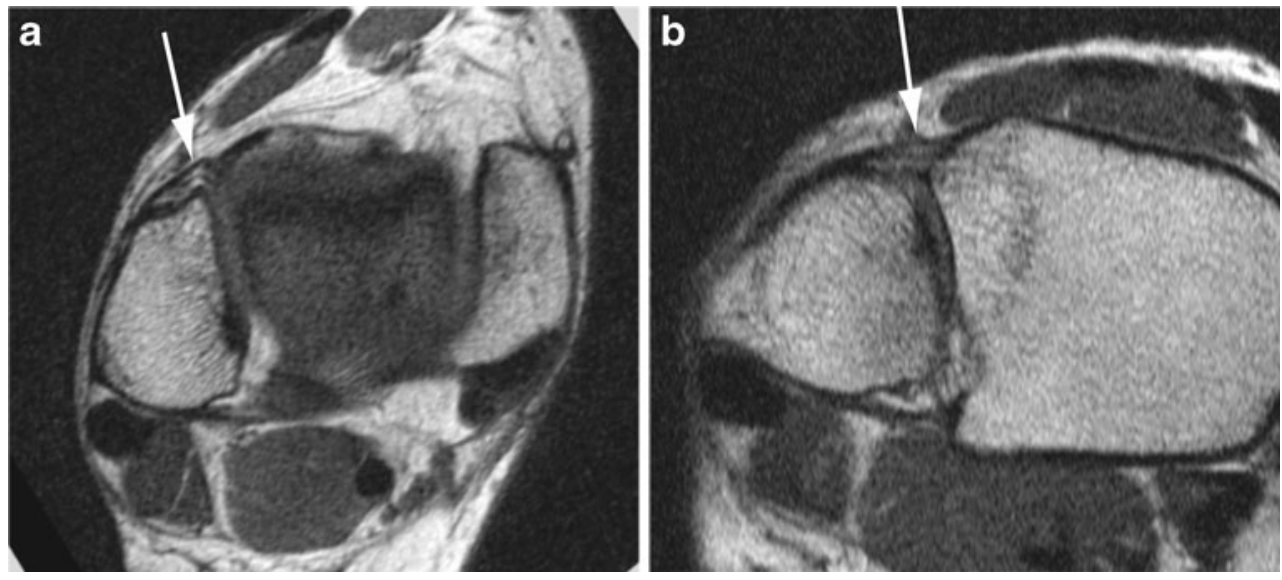
properties as an index for measuring agreement. With regard to continuity and the wavy aspect of the PTIFL in the oblique plane, the value for kappa could not even be determined [33].

We compared the oblique plane only with the axial plane and not with the coronal plane. The oblique and axial planes differ in only one parameter, i.e., the angle in which the ligaments are depicted, while the cross-sectional direction is the same; the oblique and coronal planes differ in two parameters, i.e., both in different cross-sectional directions as well as a different plane. Although the coronal view is good for depicting the multifascicular aspect of the ATIFL and PTIFL, in our volunteers the fascicles of the ATIFL and PTIFL were never entirely visible along their full length between their insertion onto the tibia and fibula. The coronal plane is therefore less suited for analysis of traumatic changes.

Our study has several shortcomings. First, we only used two cadaveric legs. It would be better to determine the range of angles of the ATIFL and PTIFL in a larger number cadaveric legs or volunteers. The ATIFL and PTIFL should perhaps each be scanned with a different optimal angle. Second, ideally, the best way to analyze the ATIFL and PTIFL would be to determine the exact angle of both ligaments in each individual separately. This would mean that in each individual first an axial and a coronal dataset should be acquired. Subsequently, in the axial plane the angle with the coronal plane could be established and next in the coronal images the angle with the axial plane. However, this would make it an elaborate and timeconsuming procedure and not very practical. We therefore chose a fixed angle of $45^{\circ}$ for the oblique MRI scan plane and accepted a small percentage (12\%) of partly interrupted ligaments. Third, although the volunteers are healthy, it is unlikely that they had never had any previous ankle trauma. On average, almost everyone sustains an inversion injury of the ankle once during their lifetime. Thirty-nine percent of the volunteers had signs of minor injury of the collateral ankle ligaments, visible as a slight thickening of one or more of the collateral ankle ligaments. Although isolated injury of the syndesmosis, known as a high sprain, can occur, it often has a more protracted recovery period and therefore would be more easily remembered. On the other hand, minor syndesmotic injury could also go undetected and heal with a slight thickening or maybe wavy aspect of the ATIFL.

Clinically, information about the integrity of the syndesmotic ligaments is of importance, because it determines the kind of therapy the patient gets. The ATIFL in particular plays an important role in the stability of the syndesmosis. In the case of an isolated injury of the ATIFL (a high sprain), a complete rupture of the ATIFL should be treated with a nonweight-bearing cast or surgery, while a partly ruptured ATIFL can be treated functionally with a weight-bearing cast.

\section{Conclusion}

The MRI scans for the syndesmotic ligaments, ATIFL and PTIFL, have been carried out in the three usual orthogonal scan planes. The advantage of scanning in an oblique image plane of about $45^{\circ}$ lies in better depiction of both ligaments compared with the axial plane, and with it a better interpretation of continuity, thickening, and wavy contour. This may lead to a reduction in false-positive results, especially regarding partly or completely ruptured ligaments, and can be of considerable aid in therapeutic management, as demonstrated by the three clinical cases.

Open Access This article is distributed under the terms of the Creative Commons Attribution Noncommercial License which permits any noncommercial use, distribution, and reproduction in any medium, provided the original author(s) and source are credited.

\section{References}

1. Cedell CA. Ankle lesions. Acta Orthop Scand. 1975;46 (3):425-45.

2. Hopkinson WJ, St Pierre P, Ryan JB, Wheeler JH. Syndesmosis sprains of the ankle. Foot Ankle. 1990;10(6):325-30.

3. Brostroem L. Sprained ankles. I. Anatomic lesions in recent sprains. Acta Chir Scand. 1964;128:483-95.

4. Gerber JP, Williams GN, Scoville CR, Arciero RA, Taylor DC. Persistent disability associated with ankle sprains: a prospective examination of an athletic population. Foot Ankle Int. 1998;19 (10):653-60.

5. Beumer A, Heijboer RP, Fontijne WP, Swierstra BA. Late reconstruction of the anterior distal tibiofibular syndesmosis: good outcome in 9 patients. Acta Orthop Scand. 2000;71 (5):519-21.

6. Beumer A, Valstar ER, Garling EH, Niesing R, Ginai AZ, Ranstam J, et al. Effects of ligament sectioning on the kinematics of the distal tibiofibular syndesmosis: a radiostereometric study of 10 cadaveric specimens based on presumed trauma mechanisms with suggestions for treatment. Acta Orthop. 2006;77(3):531-40.

7. Kelikian H, Kelikian AS. Disorders of the ankle. Philadelphia: Saunders; 1985.

8. Cedell CA. Supination-outward rotation injuries of the ankle. A clinical and roentgenological study with special reference to the operative treatment. Acta Orthop Scand 1967;(Suppl 110):3+.

9. Riede UN, Schenk RK, Willenegger H. Joint mechanical studies on post-traumatic arthrosas in the ankle joint. I. The intra-articular model fracture. Langenbecks Arch Chir. 1971;328(3):258-71.

10. Ramsey PL, Hamilton W. Changes in tibiotalar area of contact caused by lateral talar shift. J Bone Joint Surg Am. 1976;58 (3):356-7.

11. Pneumaticos SG, Noble PC, Chatziioannou SN, Trevino SG. The effects of rotation on radiographic evaluation of the tibiofibular syndesmosis. Foot Ankle Int. 2002;23(2):107-11.

12. Beumer A, van Hemert WL, Niesing R, Entius CA, Ginai AZ, Mulder PG, et al. Radiographic measurement of the distal tibiofibular syndesmosis has limited use. Clin Orthop Relat Res. 2004;(423):227-34.

13. Brage ME, Bennett CR, Whitehurst JB, Getty PJ, Toledano A. Observer reliability in ankle radiographic measurements. Foot Ankle Int. 1997;18(6):324-9. 
14. Wrazidlo W, Karl EL, Koch K. Arthrographic diagnosis of rupture of the anterior syndesmosis of the upper ankle joint. Rofo. 1988;148(5):492-7.

15. Karl EL, Wrazidlo W. Fresh rupture of the syndesmosis of the proximal ankle joint. Clinical significance and arthrographic diagnosis. Unfallchirurg. 1987;90(2):92-6.

16. Sanders HW. Ankle arthrography and ankle distortion. Radiol Clin (Basel). 1977;46(1):1-10.

17. Milz P, Milz S, Steinborn M, Mittlmeier T, Putz R, Reiser M. Lateral ankle ligaments and tibiofibular syndesmosis. $13-\mathrm{MHz}$ high-frequency sonography and MRI compared in 20 patients. Acta Orthop Scand. 1998;69(1):51-5.

18. Ebraheim NA, Lu J, Yang $\mathrm{H}$, Mekhail AO, Yeasting RA. Radiographic and CT evaluation of tibiofibular syndesmotic diastasis: a cadaver study. Foot Ankle Int. 1997;18(11):693-8.

19. Brown KW, Morrison WB, Schweitzer ME, Parellada JA, Nothnagel H. MRI findings associated with distal tibiofibular syndesmosis injury. AJR Am J Roentgenol. 2004;182(1):131-6.

20. Takao M, Ochi M, Oae K, Naito K, Uchio Y. Diagnosis of a tear of the tibiofibular syndesmosis. The role of arthroscopy of the ankle. J Bone Jt Surg. 2003;85(3):324-9.

21. Muhle C, Frank LR, Rand T, Ahn JM, Yeh LR, Trudell D, et al. Tibiofibular syndesmosis: high-resolution MRI using a local gradient coil. J Comput Assist Tomogr. 1998;22(6):938-44.

22. Vogl TJ, Hochmuth K, Diebold T, Lubrich J, Hofmann R, Stockle U, et al. Magnetic resonance imaging in the diagnosis of acute injured distal tibiofibular syndesmosis. Investig Radiol. 1997;32(7):401-9.

23. Schneck CD, Mesgarzadeh M, Bonakdarpour A, Ross GJ. MR imaging of the most commonly injured ankle ligaments. I. Normal anatomy. Radiology. 1992;184(2):499-506.
24. Bartonicek J. Anatomy of the tibiofibular syndesmosis and its clinical relevance. Surg Radiol Anat. 2003;25(5-6):379-86.

25. Oae K, Takao M, Naito K, Uchio Y, Kono T, Ishida J, et al. Injury of the tibiofibular syndesmosis: value of MR imaging for diagnosis. Radiology. 2003;227(1):155-61.

26. Kim S, Huh YM, Song HT, Lee SA, Lee JW, Lee JE, et al. Chronic tibiofibular syndesmosis injury of ankle: evaluation with contrast-enhanced fat-suppressed 3D fast spoiled gradient-recalled acquisition in the steady state MR imaging. Radiology. 2007;242 (1):225-35.

27. Beumer A. Chronic instability of the anterior syndesmosis of the ankle. Acta Orthop. 2007;78(327):4-36.

28. Grass R, Herzmann K, Biewener A, Zwipp H. Injuries of the inferior tibiofibular syndesmosis. Unfallchirurg. 2000;103(7):520-32.

29. Kapanji I. Funktionelle Anatomie der Gelenke. Gerlingen: Enke; 1985.

30. Ebraheim NA, Taser F, Shafiq Q, Yeasting RA. Anatomical evaluation and clinical importance of the tibiofibular syndesmosis ligaments. Surg Radiol Anat. 2006;28(2):142-49.

31. Bassett III FH, Gates III HS, Billys JB, Morris HB, Nikolaou PK. Talar impingement by the anteroinferior tibiofibular ligament. A cause of chronic pain in the ankle after inversion sprain. J Bone Jt Surg Am. 1990;72(1):55-9.

32. Subhas N, Vinson EN, Cothran RL, Santangelo JR, Nunley Jr JA, Helms CA. MRI appearance of surgically proven abnormal accessory anterior-inferior tibiofibular ligament (Bassett's ligament). Skeletal Radiol. 2008;37(1):27-33.

33. Feinstein AR, Cicchetti DV. High agreement but low kappa. I. The problems of two paradoxes. J Clin Epidemiol. 1990;43 (6):543-49. 